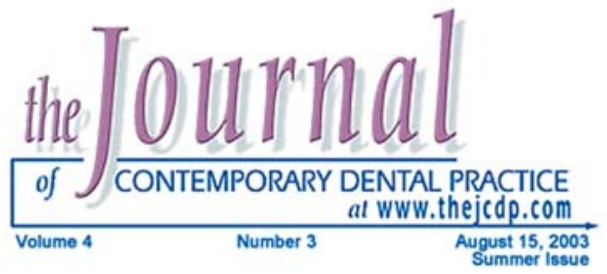

\title{
Coronal Microleakage Assessed by Polymicrobial Markers
}
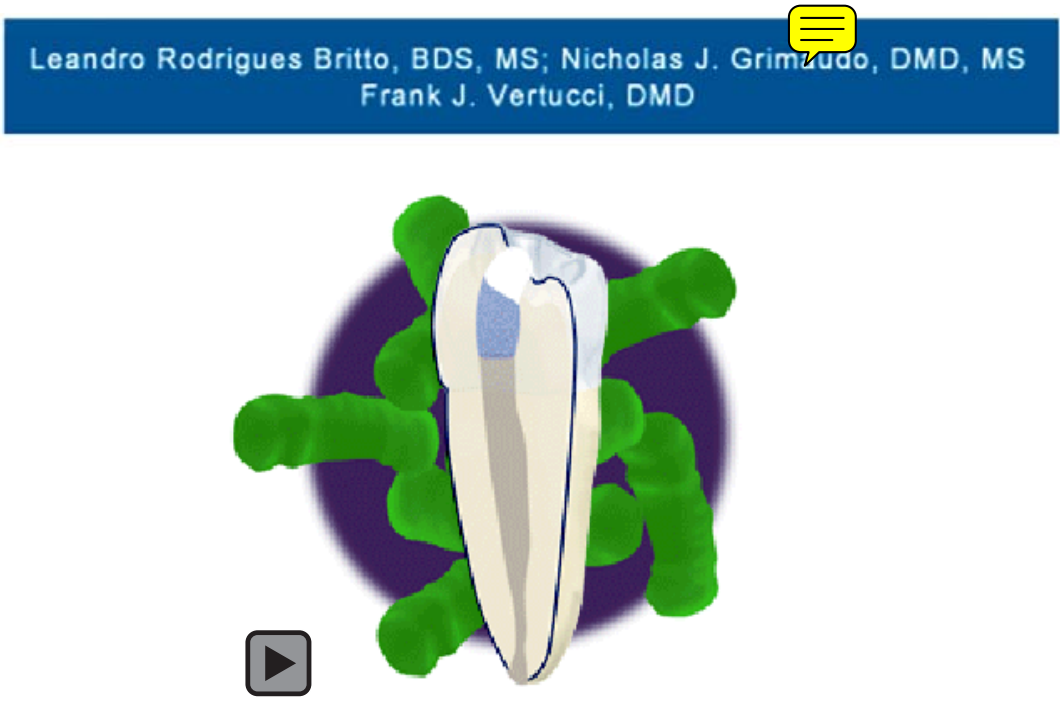

Abstract

Studies have shown significant bacterial leakage following exposure of sealed root canals to artificial and natural saliva. The objective of this study was to determine contamination via bacterial migration in artificial saliva in endodontically treated teeth using different obturation techniques and sealers. A total of 144 extracted, human mandibular anterior teeth were cleaned and shaped to a \#40 master apical file using hand and rotary instrumentation. One hundred and twenty teeth were divided into two experimental groups. The teeth in Group 1 were obturated with gutta-percha using lateral compaction and five different sealers, and the teeth in Group 2 were obturated with gutta-percha using thermomechanical compaction and five different sealers. The remaining 24 teeth were prepared as positive and negative controls. All specimens, except the negative controls, were inoculated every five days with Anaerobic streptococci (NCTC 9891) related to Peptostreptococcus micros and Prevotella intermedia (ATCC 25611). The contamination onset time was continuously recorded and turbid broths cultured for bacteria identification. The controls behaved as expected. Regardless of the combination between obturation techniques and different sealers, all broths became turbid during this experiment. The correlation between the obturation techniques and the sealers revealed statistical significance using ANOVA $(p<0.0001)$, followed by the Duncan Multiple Range Test, which determined the ranking between these interactions. The combination of MicroSeal obturation technique with Ketac-Endo sealer allowed a slower rate of coronal-apical bacterial migration.

Keywords: Coronal microleakage, artificial saliva, polymicrobial markers, endodontic therapy

Citation: Leandro Rodrigues Britto LR, Grimaudo NJ, Vertucci FJ. Coronal Microleakage Assessed by Polymicrobial Markers. J Contemp Dent Pract 2003 August;(4)3:001-010.

(c) Seer Publishing 
Introduction

Microbial contamination of the root canal system plays a significant role in reducing endodontic prognosis. ${ }^{1}$ More notable is the fact these microorganisms and their by-products frequently engender an immunologic response.

The importance of the coronal seal following completion of root canal therapy has increasingly been recognized in the dental literature as an important factor in endodontic success. ${ }^{2,3,4}$ Root canals obturated in vitro by the lateral compaction technique, when exposed to bacteria, have also been shown to become completely contaminated in thirty days. ${ }^{5}$

The concept that root canal treatment may be unsuccessful because of coronal leakage is not novel. In 1961, a study using a radioactive tracer showed coronal leakage occurred regardless of the existence of a coronal dressing. ${ }^{6}$ This study also explored the outcome of cases presenting inadequate obturation, but clinically suitable coronal seal, which in due course showed clinical and radiographic treatment failure.

Various aerobic and facultative bacteria species have been used to determine leakage in endodontics, ${ }^{7,8}$ but only a few studies have been performed with polymicrobial markers, particularly with microorganisms frequently found in endodontic related pathologies.

Fabricius et al. ${ }^{9}$ performed a coronal microleakage experiment in vivo where they inoculated 75 root canals of monkeys with 11 bacterial species separately or in combinations. They concluded mixed infections have a greater ability to induce periradicular lesions than mono infections.

The aim of this study was to develop an in vitro model that would facilitate an analysis of various combinations of sealers and/or obturation techniques to determine if any could either delay the progress of contamination or eliminate it altogether.

\section{Material and Methods}

\section{Preparation of Teeth}

One hundred and forty-four intact, caries-free human mandibular incisors with straight roots were selected for this study. No data regarding age, sex, or reasons for extraction were available. The teeth were radiographed to show the bucco-lingual dimension; those with calcified, multiple canals, or obstructions were eliminated. Specimens were handled aseptically throughout the experiment and stored in separated vials containing sodium ozide at $0.05 \%$. Preliminary results showed negative bacterial growth after specimens had been sterilized with $0.05 \%$ sodium ozide.

Roots were examined under a 60-power dissection microscope, and those with fractures were discarded. To maintain uniformity of the root canal length, the crown and some coronal root structure of each specimen was cut to obtain a root length between 13 and $15 \mathrm{~mm}$. The process of canal access, cleaning, and shaping was standardized for all specimens. The working length was established by placing an ISO size 10 stainless steel hand file (Maillefer, Ballaigues, Switzerland) into the canal until its tip could be seen at the apex and $1 \mathrm{~mm}$ was then subtracted from this measurement. The root canals were prepared by using K-files (Maillefer, Ballaigues, Switzerland) and ProFile .04 taper (Dentsply Tulsa Dental, OK, USA) rotary instruments. Apical preparation was done at the established working length to an ISO size 40 file. The coronal portions of the canal were flared with Gates-Glidden burs ISO size 070 and 090. Recapitulation with an ISO size15 file to the apical foramen was done after each larger size file was used. During the cleaning and shaping procedures, the root canal was irrigated with $2 \mathrm{cc}$ of $5.25 \% \mathrm{NaOCl}$ in combination with $17 \%$ EDTA to remove the smear layer. The canal was finally dried with a sterile paper point. Table 1 Shows the summary of the experimental and control groups.

All specimens had their access openings sealed with a sterile cotton pellet and $3.5 \mathrm{~mm}$ of IRM (Caulk Dentsply, Milford, DE, USA) and placed in a humidor for 48 hours at $370 \mathrm{C}$ to allow sealer to set. 
Table 1.

\begin{tabular}{|c|c|c|}
\hline \multicolumn{3}{|l|}{ Experimental Group 1: } \\
\hline Number of Teeth & Obturation Technique & Sealer \\
\hline 12 & Lateral Compaction & Roth's 801 \\
\hline 12 & Lateral Compaction & $\mathrm{AH}-26$ \\
\hline 12 & Lateral Compaction & Thermaseal plus \\
\hline 12 & Lateral Compaction & Sealapex \\
\hline 12 & Lateral Compaction & Ketac-Endo \\
\hline \multicolumn{3}{|l|}{ Experimental Group 2: } \\
\hline Number of Teeth & Obturation Technique & Sealer \\
\hline 12 & MicroSeal & Roth's 801 \\
\hline 12 & MicroSeal & $\mathrm{AH}-26$ \\
\hline 12 & MicroSeal & Thermaseal plus \\
\hline 12 & MicroSeal & Sealapex \\
\hline 12 & MicroSeal & Ketac-Endo \\
\hline \multicolumn{3}{|l|}{ Negative Controls: } \\
\hline Number of Teeth & Obturation Technique & Sealer \\
\hline 6 & Lateral Compaction & Roth's 801 \\
\hline 6 & MicroSeal & Roth's 801 \\
\hline \multicolumn{3}{|l|}{ Positive Controls: } \\
\hline Number of Teeth & Obturation Technique & Sealer \\
\hline 12 & Single Cone & None \\
\hline
\end{tabular}

Bacterial inoculation was started in Group 3, since they did not have any sealer inside the root canal system. After the time in a humidor, the cotton pellet and the IRM were removed from all the specimens in the experimental Groups 1 and 2. Teeth in Group 3 had their coronal access permanently sealed with IRM.

\section{Preparation of Specimens}

The coronal portion of the root canals of each tooth was connected with the cut end of a $1.5 \mathrm{ml}$ polypropylene Eppendorf tube (Fisher Scientific Equipment, Philadelphia, PA, USA) using cyanoacrylate glue and sticky wax to prevent leakage at the connection. The teeth and tubes at the connection were covered entirely with two layers of nail varnish (Max Factor, Procter \& Gamble, Cosmetics and Fragrances, Weybridge, Surrey, UK) except the apical $2 \mathrm{~mm}$ of the root. The 12 teeth used as negative controls were covered completely with two layers of nail varnish including the apical portion of the tooth.

The leakage apparatus for each specimen was set up in a laminar flow hood (MDH, Andovac,
UK) (Figure 1). The polypropylene tubes were attached to a screw top, which in turn was placed onto a $30 \mathrm{ml}$ sterile polypropylene bottle. Aliquots of $0.5 \mathrm{ml}$ Fusayama artificial saliva were injected into the polypropylene tube (coronal chamber). Aliquots of $15 \mathrm{~mL}$ of Brain Heart Infusion Blood (BHIB) plus cysteine hydrochloride were placed in the $30 \mathrm{~mL}$ bottle (apical chamber) to ensure the apical portion of the root was placed in liquid.

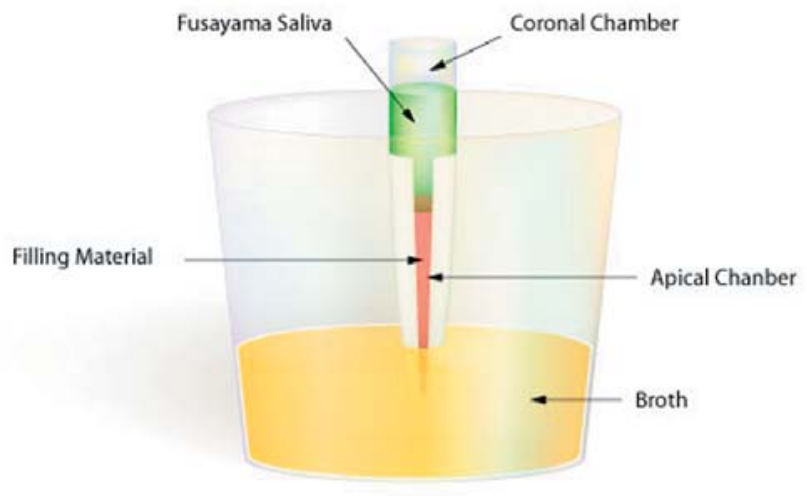

Figure 1. 


\section{Bacterial Inoculation}

The rationale for inoculating the specimens with wide-open access was to simulate loss of coronal seal and consequently initiation of the contamination process. The microorganisms used in this study were selected because of their clinical relevance, since a previous study determined that Prevotella intermedia and Peptostreptococcus spp were associated with symptomatic root canals. $^{10}$

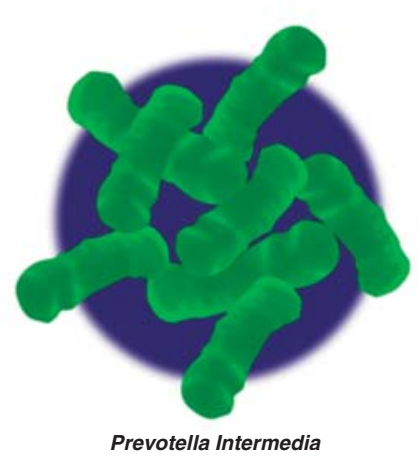

Prevotella Intermedia

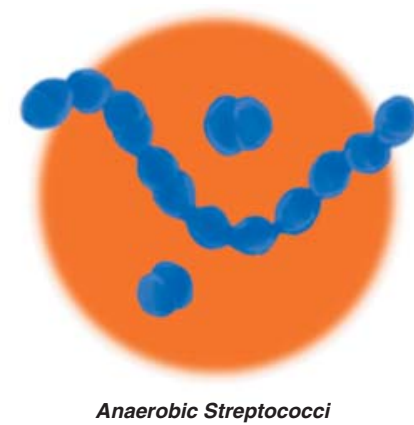

Anaerobic Streptococci

Using a sterile syringe and a disposable 22 gauge needle, the coronal chamber of each specimen was inoculated with $0.5 \mathrm{ml}$ of BHIB containing approximately $2 \times 109$ bacteria $\mathrm{ml}$ Anaerobic streptococci (NCTC 9821) related to Peptostreptococcus micros and Prevotella intermedia (ATCC 25611). The discarded broth was plated on enhanced blood agar plates (supplemented with $7.5 \% \mathrm{v} / \mathrm{v}$ debrifinated horse blood and $1 \% \mathrm{v} / \mathrm{v}$ vitamin $\mathrm{K} /$ haemin solution) to check for the presence of bacteria and possible coronal contamination. The model system was stored in an incubator (Don Whitely Scientific, Shipley, Yorkshire, UK) at $37^{\circ} \mathrm{C}$ and any changes in opacity of the broth in the apical and checked for 60 days.

Aliquots of $0.5 \mathrm{ml}$ Fusayama artificial saliva were injected into the coronal chamber and inoculated with freshly cultured microorganisms every fifth day after initial inoculation. The turbidity of the broth in the apical chamber was the first indication of contamination by microorganisms, although turbid broth had to be cultured on an enhanced agar plate to identify the microorganisms responsible for causing the turbidity. Turbid broths contaminated with microorganisms other than the ones introduced into the coronal chamber were discarded. The contamination onset time was recorded as an indicator of entire root canal recontamination.
Results

All of the positive control teeth exhibited bacterial leakage as evidenced by turbidity in the apical chamber within 48 hours, while the apical chamber of negative control teeth remained uncontaminated throughout the test period. Cultures from the positive control teeth confirmed the presence of both microorganisms on the enhanced blood agar plates. The onset time of contamination was continuously recorded and turbid broths cultured for bacteria identification. The correlation between the obturation techniques and the sealers revealed statistical significance using the analysis of variance (Super ANOVA, Abacus Concepts, Berkeley, CA, USA) $(p<0.001)$, followed by the Duncan Multiple Range Test, which determined the ranking between these variations. The MicroSeal obturation technique in combination with the Ketac-Endo sealer prevented bacterial migration the longest using this research model.

\section{Discussion}

The main advantage of the present experimental set-up over previous models is the coronal chamber was completely isolated from the apical chamber, reducing the chance for cross-contamination. The strategy used in this study proved to be a feasible and an effective method for researchers interested in studying coronal microleakage using microorganisms in vitro. However, as stated previously, after the coronal seal is lost from the root canal treated tooth, it is just a matter of time for contamination of the entire length of the root canal system by microorganisms or its by-products to take place. The amount of leakage that constitutes clinical failure has not yet been determined.

Ideally, the root canal filling should be a complete, homogenous mass that fills the prepared root canal completely. Failure to achieve this goal has been the focus of criticism of the lateral compaction technique unless the applied sealer softens the material and allows for a chemical welding of the gutta-percha cones in the canal.

The broth in all of the positive control teeth showed turbidity within 48 hours, demonstrating the microorganisms were able to penetrate the entire length of root canals filled with a single cone and no sealer. This is supported by many studies, which also showed that teeth obturated solely with gutta-percha exhibited extensive leakage. ${ }^{11,12}$ Sealers are evidently necessary. 
MicroSeal performed better than lateral compaction using this model, which means it allowed a slower rate of bacterial migration from the coronal aspect apically. One could say that MicroSeal, for being a thermomechanical compaction technique, may have provided a better root canal seal with a smaller gutta-percha-sealer-dentine interface.

A 1997 study by Wu, et al. supports our results when comparing the combination of MicroSeal- Ketac-Endo with the other combinations. ${ }^{13}$ They developed a model to study the distance between gutta-percha and the root canal wall. Using

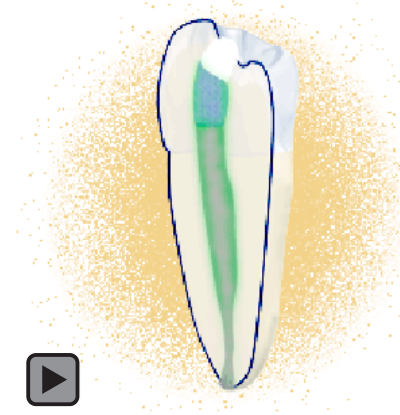
a fluid model, leakage of $\mathrm{AH}-26$ and Ketac-Endo with injected gutta-percha was measured. They reported that after condensation, the distance between gutta-percha and the root canal wall was mostly 25 microns. AH- 26 had a film thickness of 39 microns and leaked more than Ketac-Endo $(p<0.05)$ with a film thickness of 22 microns.

They concluded the sealer's film thickness is an influencing factor in the sealing quality of a root canal filling when condensation of warm guttapercha is performed. It is evident in our results that most of the combinations which included MicroSeal with various sealers showed better results when compared with lateral compaction, most likely due to this lower film thickness.

However, Ketac-Endo sealer may also have performed better than the other sealers because of its ability to adhere to the canal walls and to release fluoride. The present study demonstrated the MicroSeal obturation technique in combination with the Ketac-Endo sealer showed the greater resistance to leakage.

Additional research is not only needed to identify new methods that could prevent coronal leakage but also to recognize the microorganisms involved. This could provide practitioners with a more appropriate rationale to eliminate the etiological factor and to support the patient's immune system, particularly in retarding or even preventing recurrent infections.

\section{Conclusion}

Neither lateral compaction of gutta-percha, nor the MicroSeal Technique used with five different sealers prevented coronal ingress of a mixed microbial load in association with artificial saliva.

All turbid broth cultured in our research always revealed the presence of both microorganisms, Anaerobic streptococci and Prevotella intermedia. It was concluded the artificial saliva slowly migrated into the obturated canal through an infiltration pathway initially then the inoculated non-motile microorganisms subsequently followed this track.

The results of this study indicate the combination of Ketac-Endo sealer with the MicroSeal Technique provides superior resistance to sealer solubility and bacterial migration when compared with other combinations of sealers and obturation techniques. Nevertheless, one should take into consideration this experiment was conducted in vitro with its inherent limitations and, therefore, clinical extrapolation should be avoided.

\section{References}

1. Ingle JI, Taintor JF. Endodontics, 3rd edn. Philadelphia, PA, USA, Lea and Febiger, 1985.

2. Swanson K, Madison S. An evaluation of coronal microleakage in endodontically treated teeth. Part I. Time periods. J Endod. 1987 Feb;13(2):56-9. No abstract available.

3. Madison S, Swanson K, Chiles SA. An evaluation of coronal microleakage in endodontically treated teeth. Part II. Sealer types. J Endod. 1987 Mar;13(3):109-12. No abstract available.

4. Nguyen NT. Obturation of the Root Canal System. In: Cohen S, Burns RC, ed. Pathways of the Pulp, 6th edn, 1994; pp.219-271, St. Louis, USA: Mosby.

5. Magura ME, Kafrawy AH, Brown CE Jr, et. al. Human saliva coronal microleakage in obturated root canals: an in vitro study. J Endod. $1991 \mathrm{Jul} ; 17(7): 324-31$.

6. Marshall FJ, Massler M. The sealing of pulpless teeth with radioisotopes. J Dent Med. 1961; 16: 172-84. 
7. Lin LM, Skribner JE, Gaengler P. Factors associated with endodontic treatment failures. J Endod. 1992 Dec;18(12):625-7.

8. Gomes BP, Drucker DB, Lilley JD. Associations of specific bacteria with some endodontic signs and symptoms. Int Endod J. 1994 Nov;27(6):291-8.

9. Fabricius L, Dahlen G, Holm SE, et. al. Influence of combinations of oral bacteria on periapical tissues of monkeys. Scand J Dent Res. 1982 Jun;90(3):200-6.

10. Gomes BP, Drucker DB, Lilley JD. Positive and negative associations between bacterial species in dental root canals. Microbios. 1994;80(325):231-43.

11. Grossman, L. Antimicrobial effect of root canal cements. J Endod. 1980 Jun;6(6):594-7. No abstract available.

12. Kerekes $\mathrm{K}$, Rowe $\mathrm{AH}$. Thermo-mechanical compaction of gutta-percha root filling. Int Endod J. 1982 Jan;15(1):27-35. No abstract available.

13. Wu MK, De Gee AJ, Wesslink PR. Leakage of AH26 and Ketac-Endo used with injected warm gutta-percha. J Endod. 1997 May;23(5):331-6.

\section{About the Authors}
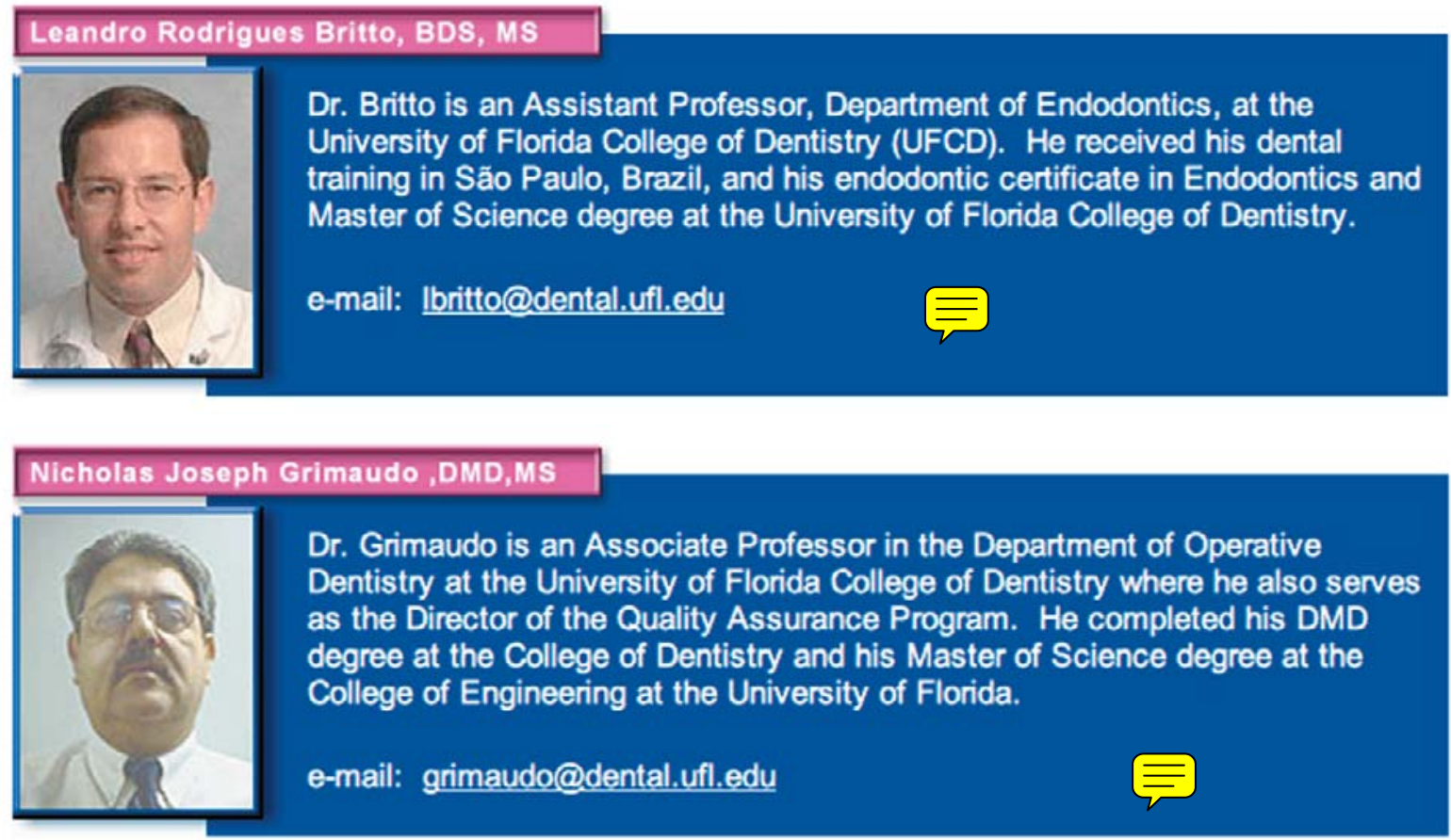

\section{Frank Joseph Vertucci, DMD}

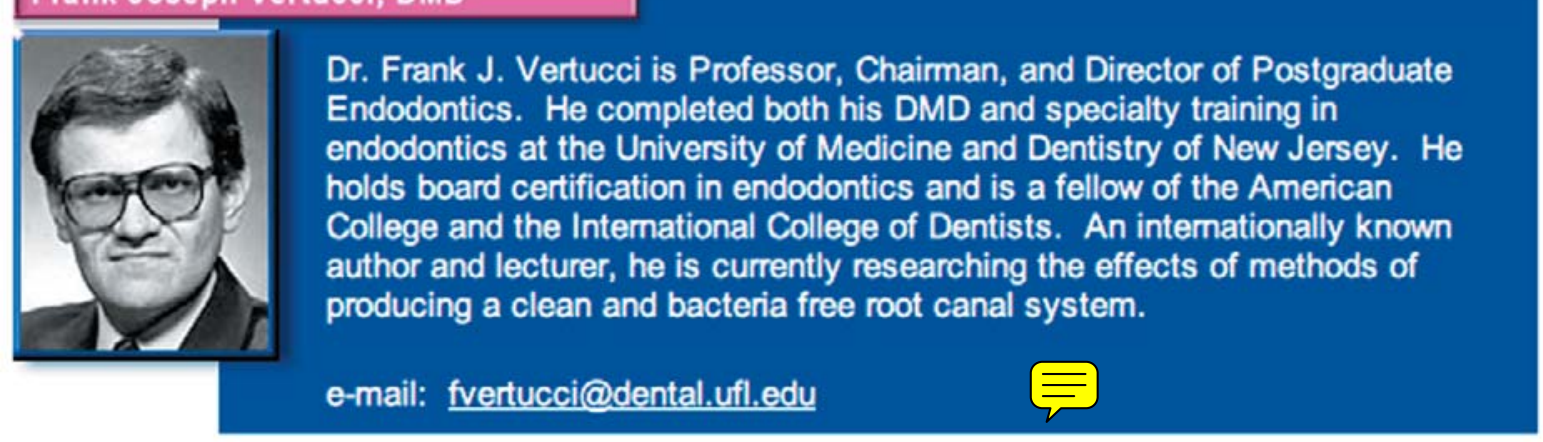

